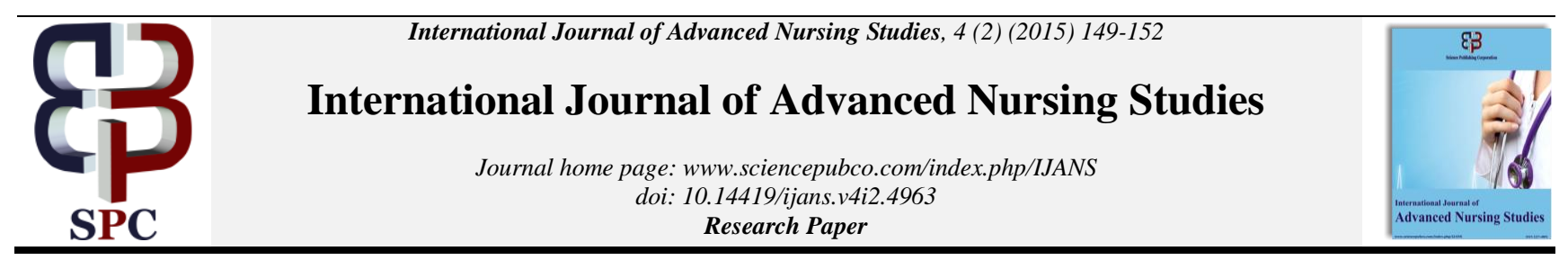

\title{
The remote area nurse model of consultation
}

\author{
Sue Lenthall ${ }^{1}$, Sabina Knight ${ }^{2}$, Sally Foxley ${ }^{1}$, Vicki Gordon ${ }^{3}$, Terrie Ivanhoe ${ }^{4}$, Robyn Aitken ${ }^{5}$ \\ ${ }^{1}$ Centre for Remote Health, Australia \\ ${ }^{2}$ Mt Isa Centre for Rural and Remote Health, Australia \\ 3 Aboriginal Medical Service Alliance of the Northern Territory, Australia \\ ${ }^{4}$ Nganampa Health Council, Australia \\ ${ }^{5}$ Northern Territory Department of Health, Australia, Flinders University \\ *Corresponding author E-mail: Sue.Lenthall@flinders.edu.au
}

\begin{abstract}
Registered nurses working in remote areas of Australia are often called remote area nurses (RANs). RANs have traditionally used models of client consultation designed for acute presentations and episodes of care. However, presentations to health care facilities in remote Australia are more likely to be chronic, complex, multi-system and multifactorial in origin and subsequent management. This paper describes a consultation model developed from a combination of expert opinion, literature and trial and feedback from RANs. The model is comprehensive, systematic and puts the person at the centre of care. It aims to mitigate risk for the client; the RAN and the health service while at the same time building trust and health literacy between the client and the RAN to encourage the client to continue with the partnership in care.
\end{abstract}

Keywords: Remote Area Nurses; Consultation; Advanced Practice; Model of Consultation; Indigenous Health.

\section{Introduction}

Nurses working in Remote Areas of Australia are known as Remote Area Nurses (RANs). RANs play an important role in the delivery of health care to communities in remote Australia, (Yuginovich 2000) with over eighty percent (80\%) working within isolated, community settings. (Lenthall, Wakerman et al. 2011). RANs provide health care across the lifespan, usually with small local teams of Indigenous Practitioners, community members, other RANs and sometimes a resident general medical practitioner (GP). Medical specialists, non-resident GPs, allied health staff and community liaison personnel support and advise the RAN and local team via telephone and/or regular visits (Lenthall, Wakerman et al. 2009) .

The client demographics and burden of disease in remote areas of Australia is markedly different to that of urban areas. There is a high percentage of Indigenous peoples in remote Australia and the general measures of disadvantage, early onset high morbidity, comorbidity and mortality rates of this group have been well recognized (Australian Human Rights Commission 2008, Australian Institute of Health and Welfare 2008). The skills and knowledge required by nurses to work effectively and safely in remote health must include; primary health care principles, cultural safety and respect and advanced clinical skills (Lenthall, Wakerman et al. 2009).

\section{Main body}

\subsection{Consultation models}

The predominant model of history taking and assessment many RANs have previously used has been guided by 'SODA-F' or 'SOAPIE'.

'SODA F' and 'SOAPIE' provide a framework for obtaining a history, conducting an examination, and documenting care in the acute-care setting. They are clinician centered, focus on the disease or problem, and are premised on the traditional medical model of client care. These models assume disease is fully accounted for by deviations from the norm of measurable biological (somatic) variables (Engel,1977 cited in Stewart (Stewart, Brown et al. 1995). Neither framework considers the social, psychological, behavioural, environmental and population health dimensions of illness.

SODA F

Subjective/Story

Objective /Observations

Diagnosis

Action

Follow-up

\section{SOAPIE}

Subjective /Story

Objective /Observations

Assessment

Plan

Implementation

Evaluation

\subsection{Constructing an alternative}

Over ten years ago a group of experienced remote health professionals teaching within a post-graduate course for RANs became aware of the inadequacy of the current models of consultation and sought an alternative model to teach and practice. The model of consultation presented in this paper is the result from a process of discussion, adoption of sentinel works from clinicians working in urban general practice clinics and extensive testing and refinement by RANs working in remote communities throughout Australia. 


\subsection{General practice consultation models}

Since the 1970s there has been a shift by health professionals including General Practitioners (GPs) to provide holistic, comprehensive care and health promotion techniques that support clients to be actively involved in their care. In 1979, Stott and Davis described "The exceptional potential in each primary care consultation" and suggested that the presenting complaint, ongoing health concerns, health promotion and health seeking behaviour be explored each time a client consults with a health practitioner (Stott and Davis 1979). Pendleton et al (1984) built upon the work by Stott and Davis (1979), describing seven tasks to undertake within the consultation. These tasks emphasized the importance of understanding the client's ideas, concerns and expectations and highlighted that management should be negotiated, collaborative and consider the use of time and resources. Stewart et al (1995) took this concept further to encourage the practitioner to find out not just what the client wanted or was concerned about but to explore their experience of the illness and its impact on them. (Neighbour 2005) The former developed the notion of team building and use of resources by promoting the importance of general practice 'housekeeping' and GP self-care within the consultation model.

\subsection{RAN model of consultation}

The RAN model developed from a combination of expert opinion, literature and trial and feedback from RANs. It represents a systematic comprehensive approach to each client consultation and has seven principles and eight steps.

\subsection{RAN model of consultation - principles}

Culturally safe approach, consider:-

- Who is the right person to do the consult, gender issues?

- How can I make the person feel comfortable?

- Is there need for an interpreter?

- Are appropriate family members present if necessary?

Holistic and comprehensive

- Considers the whole person - the context of their lives, their families and community.

- Guided by population health principles.

- Explores the client's experience of their illness and how it impacts on them and their therapeutic goals.

- Considers chronic diseases /on-going health problems/age appropriate screening.

- Guided by context and environment.

Systematic

- History before observations or the physical examination. The history informs the examination.

Shares power with the client

- Based on partnership between the RAN, the client and heath service.

- Negotiates with the client around history taking and management plan.

- Encourages the client to share in the decision making and own their own health.

- Assists with building the client's self-reliance and health literacy.

Provides coordination and continuity of care

- Recognises share care.

- Includes succinct, pertinent client record notes.

- Provides summaries to nominated services, coordinates complex care.

- Collaborates with colleagues.

- Activates recall.

Encourages clinical reasoning

- Considers the age /place risk of the client, what it is most likely, what can't you afford to miss.

- Uses a problem solving approach in order to reach a hypothesis.
Promotes clinical safety and quality

- Work within RANs own individual scope and context of practice.

- Utilises endorsed best practice treatment protocols and quality improvement processes and procedures.

\subsection{RAN model of consultation - steps}

1) Open consultation

Before seeing the client

- Review clinic records, note outstanding recalls or actions, ensure privacy

When seeing the client

- Greet client and establish rapport

- Note general impressions

- Check name, next of kin, chart number and DOB are correct

- Check if interpreter or practitioner of other gender needed

2) History

Reason for presentation Acute / Chronic disease review / Screening

- Listen to story, establish ideas, concerns and expectations,

- If acute - OLDCARTS for each reason for presentation

- Ask questions relevant to system involved

Current Health Review

- Appetite, nausea, change in weight, sleep, energy/activity, bowels, 'waterworks', interest in life, periods, sexual health, emotional health - self harm, "do you ever feel unsafe?"

- Drug/allergy history: medications (prescribed, over the counter and traditional), allergies and immunization status

- Alcohol/other drugs / smoking

Other history

- Past Medical/surgical history: Accidents, admissions to hospital, treatments at other clinics, accidents and injuries, chronic diseases, Gynaecological / obstetric history including last Pap smear

- Family medical history: general health

- Personal and social history

3) Clinical examination

- Observation of appearance / gait etc.

- Rapid screening exam

- Vital signs, Urinalysis, $\mathrm{SaO}$, Blood glucose, $\mathrm{Hb}, \mathrm{BMI}$

- Systematic assessment of relevant systems - LOOK LISTEN FEEL

- Investigations: as indicated by history and age/place/ risk for screening and patient consent

4) Assessment and discussion

Make an assessment of the reason for presentation and of other health issues

- Summarise and reflect findings from the history and clinical examination with the client and discuss assessment

- Seek further advice as needed

- Explore the person's knowledge and clarify - including long and short term implications.

- Assess and use opportunity for brief intervention and health promotion as appropriate

5) Negotiate a management plan

- Follow best practice treatment manuals

- Discuss goals and targets, negotiate management with the client and appropriate others

- Refer as necessary for further investigation or management

- Include when appropriate long term care, identified risk factors, public health screening and preventative health

6) Close consultation 
- Check client's understanding and agreement of management plan- questions?

7) Documentation

- Update client's record using SOAP, progress notes and clinical items, recall system and SEHR

\section{8) Reflection}

- What went well? What could be improved on? How are you?

\subsection{Advantages}

The RAN consultation model is comprehensive, systematic, safe and puts the person at the centre of care. The model encourages RANs to respond to clients and be sensitively proactive. Modern clinical practice requires RANs to deal with the presenting complaint often acute and consider ongoing health problems usually chronic, the potential interaction between these problems and social, cultural and environmental factors. Such a proactive approach includes undertaking adult or child health checks, implementing health promotion and prevention activities, which save time and resources when offered and undertaken opportunistically, even if completed in full in subsequent visits. It is poor clinical practice that a child with long standing anaemia and associated poor growth may only be treated for several episodes of infection despite multiple presentations, or present years later as an adult with end-stage renal failure secondary to undetected and untreated childhood renal insufficiency.

The model is also protective against risk to the client, the RAN and the health service. High levels of morbidity and mortality and their consequences must be taken seriously in remote Australia. The potential for rare clinically significant conditions such as meningitis, melioidosis or common fewer visible conditions such as pelvic inflammatory disease or renal failure progression must be considered. The adage of 'what can't I afford to miss?' guides clinical decision making and is underpinned by knowledge of the population and the geographical region. A willingness to ask is essential, and the model encourages this curiosity. Discussing the findings of the consult with the client is another key component of the RAN model of consultation and allows clarification of issues and opportunities to reduce risk by clarifying information, implementing health promotion initiatives, age and gender appropriate screening and brief interventions. The model encourages RANs to be mindful of limits to their individual professional capability, context and to refer or seek advice from colleagues and medical officers appropriately. Respectful and mutually supportive collegial relationships, as well as reliable and effective communication are highlighted as essential.

High turnover of staff means the client may see many providers. Combined with RANS usually providing after hours on "call care", safety and continuity of care are both an issue. The risk of fatigue is high hence relying on a trusted comprehensive consultation model provides safety for both the RAN and client.

The quality of the interaction between the RAN, health service ,the client, and sometimes the family will determine the confidence, trust and willingness of the client to continue with the partnership of care. An effective strategy for achieving this objective is the negotiation of management and health plans. Partnership is a central feature to the model. The model provides a framework for the RAN and the client to identify together important clinical issues, health goals and activities, the options for care, the pharmacological and supportive therapy options, and limitations and logistical challenges. This negotiated partnership process contributes to improved therapeutic relationship and optimises the compliance potential with treatment regimes. When using the model, each encounter aims to build the client's understanding of their health, their illness or risk and their confidence to make and articulate decisions about their own life and care. With chronic disease the RAN, model of consultation provides the client with the opportunity to become the expert, while the RAN adopts the role of providing new information and coordinating care over time.

\section{Conclusion}

The RAN model of client consultation has been developed to assist RANs fulfil their complex and diverse role serving the needs of people living in remote and Indigenous communities. The model was driven by a morbidity shift from infectious and acute illnesses to also include the current chronic disease epidemic with infectious disease and trauma. The model has been trialled and refined over a period of ten years by RANs across Australia. The RAN model of client consultation enables safe consultations with clients in remote primary health care. With Indigenous clients, it follows the principles of cultural respect. The RAN model of consultation provides a model for nurses in remote and Indigenous settings to systematically work through steps of establishing a relationship with the client in order to understand the whole person, their experience of illness and aspirations in the context of their culture, physical environment, their family and the community in routine clinical work or after hours when tired and on call; By using the model, the RAN can make an assessmentand/or disease risk and collaborate with the client and other team members to implement management. This model encourages RANs to develop improved communication skills within the health care team. In contrast to the other models currently used by nurses working in remote settings, The RAN using this model ensures that clients have improved access to timely and comprehensive health care. The model provides a framework for practice relevant to both routine and after hours consultations and has the potential to minimize some of the impact of high staff turnover on small teams. Accordingly, this model has at its heart a focus on improving health outcomes of Indigenous peoples and vulnerable populations in remote Australia.

\section{Acknowledgement}

Thank you to the nurses and Indigenous health practitioners, who have trialed this model and provided feedback to the authors, and the academics and medical practitioners who have assisted with the underpinning theoretical principles.

\section{References}

[1] Australian Health Ministers' Advisory Council - Standing Committee for Aboriginal and Torres Strait Islander Health Working Party (2004). Cultural respect framework for Aboriginal and Torres Strait Islander health, 2004-2009. S. A. Department of Health. Adelaide.

[2] Australian Human Rights Commission (2008). A statistical overview of Aboriginal and Torres Strait Islander people in Australia. Canberra, Australian Human Rights Commission.

[3] Australian Institute of Health and Welfare (2008). The_Health and Welfare of Australia's Aboriginal and Torres Strait Islander Peoples. Canberra, AIHW.

[4] Close the Gap (2008). Statement of Intent, Indigenous Health Equality Summit.

[5] Lenthall, S., J. Wakerman and S. Knight (2009). "The frontline and the ivory tower: A case study of service and professional-driven curriculum." Aust. J. Rural Health 17: 129-133. http://dx.doi.org/10.1111/j.1440-1584.2009.01056.x.

[6] Lenthall, S., J. Wakerman, et al. (2011). "The Nursing Workforce in Very Remote Australia, Characteristics and Key Issues." Aust. J. Rural Health 19: 32-37. http://dx.doi.org/10.1111/j.14401584.2010.01174.x.

[7] Lenthall, S., J. Wakerman, et al. (2009). "What stresses remote area nurses? Current knowledge and future action." Aust. J. Rural Health 17: 208-213. http://dx.doi.org/10.1111/j.1440-1584.2009.01073.x.

[8] Murtagh, J. (2011). John Murtagh's General Practice. Sydney, McGraw-Hill Medical.

[9] Neighbour, R. (2005). The Inner Consultation: How to Develop an Effective And Intuitive Consulting Style. London, Radcliffe Publishing. 
[10]Pendleton, D., T. Schofield, et al. (1984). The consultation: an approach to teaching and learning. Oxford: , Oxford University Press.

[11]Stewart, M., J. Brown, et al. (1995). Patient Centred Medicine, Transforming the Clinical Method London., SAGE Productions.

[12]Stott, N. C. H. and R. H. Davis (1979). "The exceptional potential in each primary care consultation." Journal of the Royal College of General Practitioners 29: 201-205. 